\title{
A Nurse Training Program for Robotic Skills Acquisition and Career Advancement
}

\author{
Ahmet Gudeloglu', [MD] \\ ORCID: 0000-0003-2927-9597
}

Sijo J. Parekattil ${ }^{2}$, [MD]

ORCID: 0000-0001-6847-8826

\author{
${ }^{1}$ Department of Urology, Hacettepe University Faculty \\ of Medicine, Ankara, Turkey. \\ ${ }^{2}$ Avant Concierge Urology, University of Central Florida, \\ FL, USA. \\ Corresponding Author: Ahmet Gudeloglu \\ Department of Urology, Hacettepe University Faculty of \\ Medicine, Ankara, Turkey. \\ Phone: +903123051970 \\ E-mail: a_gudeloglu@yahoo.com
}

https://doi.org/10.32552/2021.ActaMedica.511

\section{rep ABSTRACT Ceen}

Objective: Robotic surgery presents the state of the art surgical techniques in the era of minimally invasive surgery. A nurse's role in surgery has been altered with the development of robotics. Our unique program at Polk State College in Florida was a robotic nursing program in which we certified nurses after a great deal of training. In this study our goal was to assess the survey outcomes of this program and to see if there was room for any improvements.

Materials and Methods: We have successfully completed 4 threeday courses. During these courses we trained a total of 30 nurses and technicians. This special three-day course involved learning through online modules, didactic education, hands on training, and live surgery. We asked for their response to various questions about the course through an online survey. The trainees were asked to rank the questions about the program.

Results: We obtained 20 out of 30 responses from our certified trainees. Seventy-five percent of the certified trainees agree that this robotics nursing program has helped them advance in a career. Overall, 85\% of the certified trainees stated that this program was beneficial to them, and $95 \%$ agree that they would recommend this robotic nursing program to others.

Conclusion: This survey demonstrated a well-designed robotics nursetraining program might help trainees to gain robotic skills. Also, they declared that their certification helped them make some kind of advance in their career.

Keywords: Robotics, robotic surgery, nursing training, career

Received: 17 September 2020, Accepted: 23 October 2020,

Published online: 27 May 2021

\section{INTRODUCTION}

Robotic surgery presents the state of the art surgical techniques in the era of minimally invasive surgery. Although several types of robotic systems had been tried for various types of surgeries, today the da Vinci Surgical system (Intuitive Surgical Inc., Sunnyvale, $C A$ ) is the most commonly used and the first Food and Drug Administration (FDA) approved robotic system [1]. Currently, the da Vinci robot system is widely used in numerous disciplines from urology to gynecological [1]. Nearly $60 \%$ of the hospitals in the United States have at least one da Vinci system [2].
A nurse's role in surgery has been altered with the development of robotics. The nursing care that patients require is now different due to the minimally invasive procedures. Nurses also need an immense amount of education about the technology of the robot, how to assess it's impact, and how to better care for the patients [3].

Our unique program at Polk State College in Florida was a robotic nursing program in which we certified nurses after a great deal of training. This three-day program taught nurses the correct techniques and 
their role in robotic surgery. We had had certified thirty nurses and had conducted a survey in order to evaluate the effects of this program. We have sent out e-mails to each of the course participants and asked them to participate in a survey. In this study our goal was to assess the survey outcomes of this program and to see if there was room for any improvements.

\section{MATERIALS and METHODS}

We obtained an Institutional Review Board exemption for a study that retrospectively evaluates a robotic nurse-training program. We have successfully completed 4 three-day courses. During these courses we trained a total of 30 nurses and technicians.

The structure of the training program was presented in a way that will benefit each trainee and educate them with adequate knowledge about preparation of the da Vinci robotic system and management of a robotic surgery. This special three-day course involved learning through online modules, didactic education, hands on training, and live surgery. The trainees were first introduced to the basics of laparoscopy and robotic-assisted laparoscopic procedures, and then informed about robotic components, robotic setup including draping of the robot, robotic instrumentation, patient positioning, and docking of the robot. The hands on training portion of this course allowed trainees to integrate the knowledge they have gained from the online modules and to further learn about the robotic surgeries. Problem solving sessions were also incorporated to prepare trainees for unexpected situations in that they need to act quickly and logically.

The agenda ended with a written test and performance to ensure the trainee was adept with the correct amount of education. The emphasis of this special program was to properly educate nurses in the field of robotics and to prepare them for situations that may occur during these robotic surgeries.

Three months from the last course we reached out to our trainees through e-mails. We asked for their response to various questions about the course through an online survey. The trainees were asked to rank the questions about the program from 1 to 10 (1=least favorable, $10=$ most favorable). And we considered a score of 7 and higher to be favorable.

\section{RESULTS}

We obtained 20 ( 2 from males, 18 from females) out of 30 responses from our certified trainees. Mean age of the respondents was 37.7 years.

Prior to the program, $40 \%$ of the certified trainees were employed as an RN in the OR, 30\% were certified as an OR technician, 20\% were employed as a nursing student, and $10 \%$ were a $\mathrm{RN}$ in an area other than the OR. At the time during survey, $45 \%$ used to work as RNs in the OR, 30\% were OR technicians, $20 \%$ were RNs in an area other than the $\mathrm{OR}$, and $5 \%$ were nursing students. Also prior to the certification of this program, $45 \%$ of the trainees had previous robotic surgical experience, while $55 \%$ did not have any experience at all. After the program, $60 \%$ of trainees have experienced robotics at work, while $40 \%$ have not encountered any robotic experience at work.

Seventy-five percent of the certified trainees agree that this robotics nursing program has helped them advance in a career. When asked how helpful the online education materials were during the training program, $80 \%$ of trainees responded with a rate of 7 and higher. Similar to the online materials, 90\% of trainees rated the didactic education portion of this program 7 and higher. Eighty-five percent of trainees felt that the hands on training portion of this program were helpful, giving scores of 9 and 10 on the scale. Another $85 \%$ answered that the live surgery part of this program was helpful (scores of 7 and above) (Figure 1).

Overall, $85 \%$ of the certified trainees stated that this program was beneficial to them, and $95 \%$ agree that they would recommend this robotic nursing program to others (Figure 2). Prior to the program, $70 \%$ of the trainees felt somewhat uncomfortable setting up and handling the robotic platform. After the completion of the program, $100 \%$ of trainees responded that they felt comfortable with setting up and handling the robotic platform. 


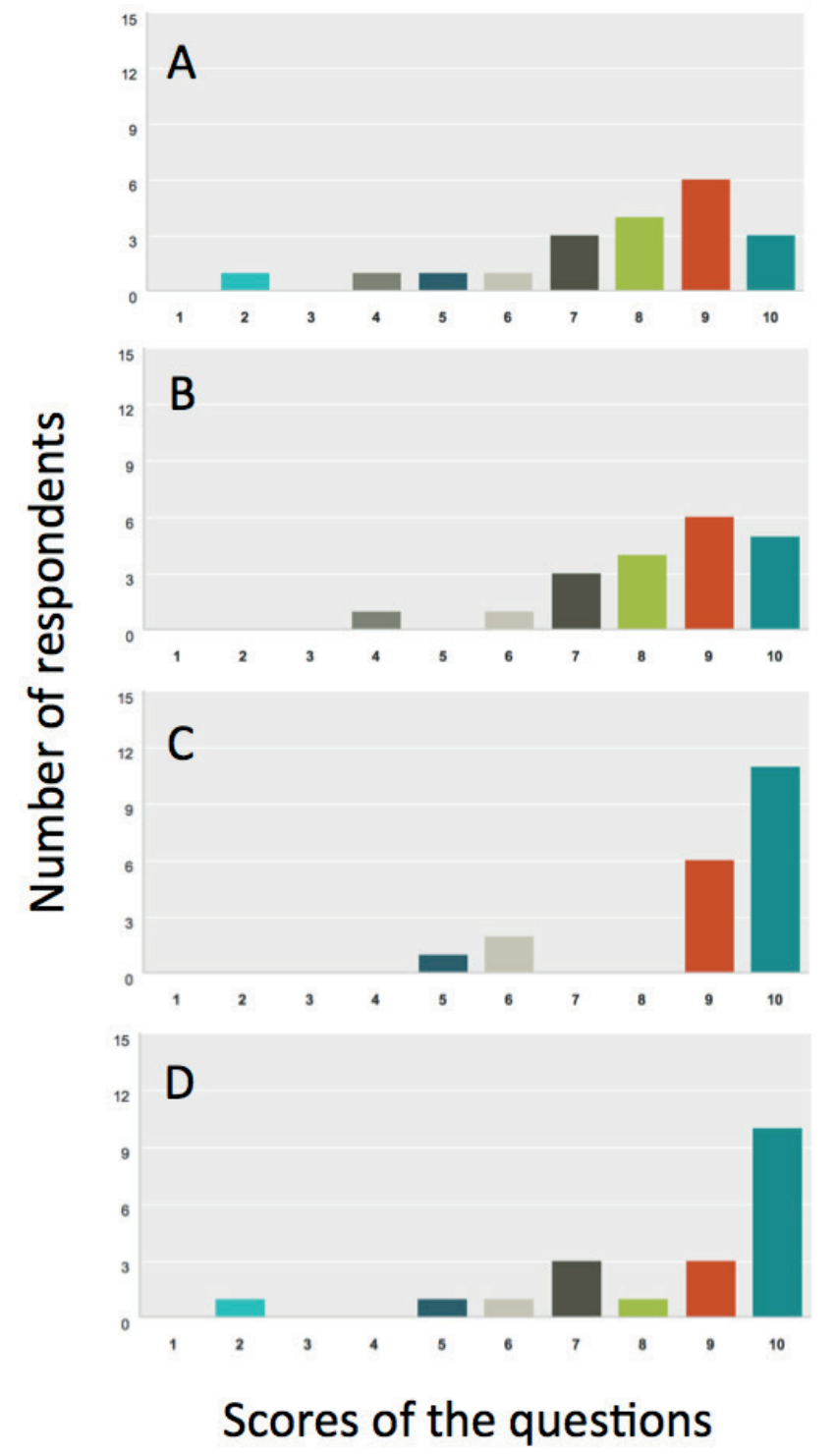

Figure 1. Trainees rank about the program. A:Online Education Materials. B: Didactic Education. C: Hands-on Training. D: Live-Case. (rate from 1-10, 1:not at all, 10:very)

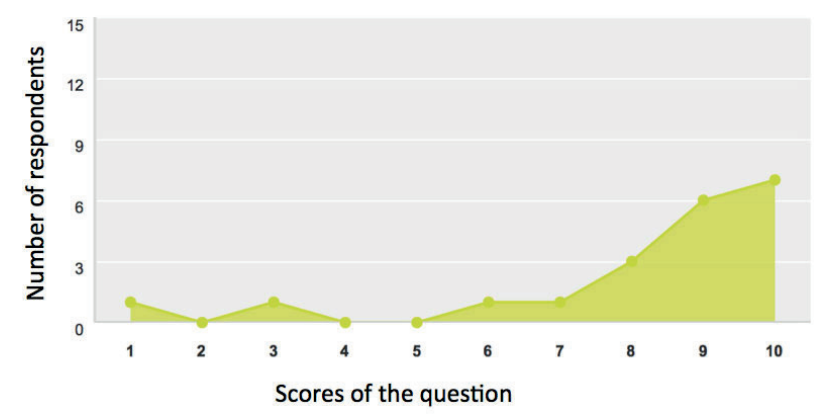

Figure 2. Trainees rank about overall how helpful the program. (rate from 1-10, 1:not at all, 10:very)

\section{DISCUSSION}

A robotics nurse needs to have the correct skills to do many new tasks. Some of these tasks include: identifying the system's components and know how they are used, prepare the system prior to surgery, know emergency procedures, know how to clean the robot correctly, troubleshoot error messages, and position the patient and equipment the right way $[4,5]$. It is beneficial to hospital facilities to have a robotics nurse specialist. This nurse specialist would be present in all procedures and would allow accurate assessments to be made of resource needs of nursing personnel and problems with the robot equipment to be solved faster and with more accuracy. This specialist would also be the ideal person to revise conflicts and bring these concerns to staff members to be solved $[6,7]$.

The job of a robotic nurse specialist includes: coordinating schedules, ensuring instrument availability, assisting with robotic procedures, proving patient and staff education, and assisting with research $[5,6]$. This job is challenging and involves an immense amount of knowledge and proficiency, but is also very rewarding and exciting. Preparing the surgical system before surgery is a crucial step in learning about the robot. The nurse needs to learn how to connect the proper cords from the video tower and the arms of the robot to the surgeon's console. Nurses also need to make sure these cords are out of the way and labeled so they are easily identifiable. The arms of the robot also need to be covered correctly with sterile drapes that hold the camera adapters. By following the manufacturer's instructions, the nurses can prepare the sterile camera and sterile scope for surgery [4]. Prior to our program, over half $(55 \%)$ of trainees did not have any kind of experience with a robot. After the program ended, $60 \%$ now encounter robotic experience at the workplace. Most respondents agree $(85 \%)$ that the hands on training portion of this program was the most helpful.

It is important that the robotic instrument cart be fully stocked at all times in case surgeons ever need extra instruments, or if the instruments malfunction. Nurses should also be aware of the location of the emergency hex wrench that comes 
with the equipment in case of a power outage or system failure. This wrench is used to free the arms of the robotic in instances like these. A nurse should always be on the lookout for any faults or errors and report these to the surgeon immediately [7]. Our unique program was designed with problem scenarios for trainees to be solved.

The position of the patient during robotic surgery is imperative and should be consulted with the anesthesia provider. Nurses should be ready to help the anesthesia provider if the patient develops extreme tracheal swelling during the procedure that could complicate extubation [4]. A nurse is also responsible for tracking how many times the robot is used and sterilizing new equipment.

A "time-out" immediately before surgery is a time where the nursing staff is able to check the patient positioning and padding. During this time, the nurses can adjust any equipment they may have not had time to do before [8]. Equipment failure can account for almost $7 \%$ of surgical alterations in some surgeries, so it is important to ensure that equipment is working correctly [8]. The timeout also gives the nurses time to inform the OR administration to ensure that there are enough specialized robotic trained nurses in the OR.
Nineteen out of twenty respondents would recommend this program to others and say that the program was interesting and very informative. Seventy-five percent of trainees say this program has helped them make some kind of advance in their career. All of the trainees now feel comfortable with the setup and handling of the robot after the program.

One of the limitations of this study is that we used online survey instead of in person survey. Also we used the da Vinci Si robotic platform that was previous generation of the da Vinci surgical systems.

\section{CONCLUSION}

This survey demonstrated a well-designed robotics nurse-training program might help trainees to gain robotic skills. Also, they declared that their certification helped them make some kind of advance in their career.

\section{CONFLICT of INTEREST}

The authors declare that there is no conflict of interest.
[1] Leal Ghezzi T, Campos Corleta O. 30 Years of Robotic Surgery. World journal of surgery. 2016;40(10):25507. Epub 2016/05/15. doi: 10.1007/s00268-016-3543-9. PubMed PMID: 27177648.

[2] Gary Guthart. J.P. Morgan Healthcare Conference 2021.

[3] Francis P, Winfield HN. Medical robotics: the impact on perioperative nursing practice. Urol Nurs. 2006;26(2):99104, 7-8. Epub 2006/05/18. PubMed PMID: 16703917.

[4] Tabor W. On the cutting edge of robotic surgery. Nursing. 2007;37(2):48-50. Epub 2007/02/03. PubMed PMID: 17273083.

[5] Yuh B. The bedside assistant in robotic surgery--keys to success. Urol Nurs. 2013;33(1):29-32. Epub 2013/04/06. PubMed PMID: 23556376.
[6] Francis P. Evolution of robotics in surgery and implementing a perioperative robotics nurse specialist role. AORN journal. 2006;83(3):630-42, 44-6, 49-50 passim. Epub 2006/04/04. PubMed PMID: 16579121.

[7] Schiff L, Tsafrir Z, Aoun J, Taylor A, Theoharis E, Eisenstein D. Quality of Communication in Robotic Surgery and Surgical Outcomes. JSLS. 2016;20(3). Epub 2016/08/06. doi: 10.4293/JSLS.2016.00026. PubMed PMID: 27493469; PubMed Central PMCID: PMC4949353.

[8] Song JB, Vemana G, Mobley JM, Bhayani SB. The second "time-out": a surgical safety checklist for lengthy robotic surgeries. Patient safety in surgery. 2013;7(1):19. Epub 2013/06/05. doi: 10.1186/1754-9493-7-19. PubMed PMID: 23731776. 